Review

\title{
Modification of Carbon Nanotubes as an Effective Solution for Cancer Therapy
}

\author{
Sara Tavakolifard ${ }^{1}$, Esmaeil Biazar ${ }^{2}$ \\ ${ }^{1}$ Department of Chemistry, Science and Research, Yazd University, Iran. \\ ${ }^{2}$ Department of Biomaterials Engineering, Tonekabon Branch, Islamic Azad University, Tonekabon, Iran. \\ Corresponding author. E-mail: kia_esm@yahoo.com
}

Received: Jul. 13, 2016; Accepted: Sep. 7, 2016; Published: Sep. 15, 2016.

Citation: Sara Tavakolifard, Esmaeil Biazar, Modification of Carbon Nanotubes as an Effective Solution for Cancer Therapy. Nano Biomed. Eng., 2016, 8(3): I 44-160.

DOI: 10.5101/nbe.v8i3.p144-160.

\begin{abstract}
Carbon nanotubes (CNT) as a new class of nano-materials hold great potential for various biomedical applications. Owing to their unusual properties, carbon nanotubes have been extensively employed in electronics, nanotechnology and optics, among others. In spite of the great potential of carbon nanotubes in various domains of biomedicine, ineffcient dispersion in aqueous solutions and biological activities in vivo are still disputable. One important and feasible route in a struggle to overcome these obstacles is modification of CNTs with organic compounds and polymers, which have been widely studied and play a crucial role in biological and biomedical fields, particularly in the cancer therapy. This review focuses on the breakthrough of the recently used methods to functionalize onto the surface of carbon nanotubes with multiple chemical species in order to produce anticancer drug delivery systems for biomedical applications.
\end{abstract}

Keywords: Drug delivery; Cancer therapy; Carbon nanotubes; Biological transporters; Targeting

\section{Introduction}

It has been observed that advances in functionalization chemistry have been considered as a driving force in the development of new classes of new nanomaterials for application in biology and medicine in the past decade [1-24]. The employment of Carbon nanotubes (CNTs), with their unique physical and chemical properties, is promising in the development of new generations of anticancer drug delivery systems. Modifications of CNTs by polymers through covalent and non-covalent bonding is one of the best approaches to adapt the collaborations of the Carbon nanotubes with environmental components together with solvent and cells. Notably, CNTs have attracted considerable interests in this regard, as they offer the potential collaborations between CNTs and polymers which are important as newly made hybrid nano-materials being simply able to cross cell membranes without any adverse biological response and, furthermore they are able to deliver a wide range of biologically active molecules containing drugs and genes into the cells. CNTs offer potential advantages over the more widely studied various nanoparticle systems, their ability to carry a high cargo loading, their intrinsic stability and structural flexibility, which could prolong the circulation time and hence the bioavailability of the carried drug molecules [25-28]. Drug delivery is an 
emerging field focusing on a wide range of biologically active molecules including drugs and genes for a desirable group of cells. The goal of this targeted delivery is to transport a proper amount of drugs to the desirable sites such as diseased tissues, with minimizing unwanted side effects of the drug on other tissues. The development of targeted therapy represents an exciting and a new approach to cancer treatment, and due to their promising properties the targeted drug as a responsive molecule attached to specific surface receptor proteins located in cell membranes [29-31]. Being endowed with targeting specificity, the carrier is able to focus just on the looked-for biological area. The use of these biological carriers for the development of specific and sensitive site-targeted bio-nano-systems makes the selective internalization of molecules in cancer cells possible. This internalization process is not feasible under normal conditions [32, 33]. Hence, such bio conjugation holds remarkable potential for future cancer treatment. Bio conjugation makes a base for further studies on cancer treatment in the future. Despite excellent progress in using CNTs as drug delivery vehicles in bio functionalization of CNTs using various biological molecules such as polyethylene glycol (PEG), DNA, antibodies, folate, cytostatic drugs, and growth factors [34-37], there is a significant lack of knowledge on how to obtain selectivity of these compounds for a single type of cancer cell. This shortage is due to the simultaneous presence of the receptors used for the specific binding of the targeting molecules to the membranes of the noncancerous cells, even in smaller amount than the cancer cells [38-40]. In this review, we focus on summerizing the latest progress in the use of CNTs for covalent and noncovalent interactions with various types of biological species, as well as bio macromolecules interactions which are very crucial to descending the toxicity of CNTs in order to study the in vitro selective drug delivery of cancer cells.

\section{Physical and Chemical Properties of the Carbon Nanotube}

More allotropes of carbon have been reported and a large scientific community has been passionate about deciphering the properties of this element that can adopt many structures ranging from diamond and graphite (three-dimensional) to graphene (twodimensional), nanotubes (one-dimensional) or fullerenes (zero-dimensional). In this way, CNT is analogous to fullerenes and graphene, which vary in wall number, diameter, length and surface chemistry [41]. Carbon nanotubes were first discovered in 1991 by Sumio Iijima in Japan [41]. Carbon nanotubes are well-ordered, hollow nanostructures consisting of a hexagonal arrangement of $\mathrm{sp}^{2}$ hybridized carbon atoms (C-C distance is about $1.4 \AA$ ) which are stronger than sp and $\mathrm{sp}^{3}$ bonds and are the key factors rendering CNTs excellent mechanical strength and high electrical and thermal conductivity. Conceptually, Graphene is the two-dimensional, hexagonal $\mathrm{sp}^{2}$-carbon ring networks with one atomic layer thickness, while CNTs can be imagined to form when the graphene sheet is rolled to be held together by van der Waals into a cylinder [42, 43]. One rolled-up graphene sheet will form a single walled carbon nanotube (SWCNT), while more than one concentric graphene sheet create a multi-walled carbon nanotube (MWCNT). Single wall carbon nanotubes have diameters ranging typically from 0.5 to $1.5 \mathrm{~nm}$ and a length ranging from 100 $\mathrm{nm}$ up to several micrometers. MWCNTs have larger diameters (can be more than $100 \mathrm{~nm}$ ) due to multilayer structures. The interlayer separation of the graphene layers of MWCNTs have a larger outer diameter (2.5 to $100 \mathrm{~nm}$ ) than SWCNTs ( 0.6 to $2.4 \mathrm{~nm}$ ); additionally, SWCNTs have a better defined wall, whereas MWCNTs are more likely to have structural defects, resulting in a less stable nanostructure. Among the various types of nano-materials (that is, materials that have at least one dimension less than $100 \mathrm{~nm}$ ), there has been intense interest in the allotropes of carbon due to their unique physical and chemical properties, emerging as promising candidates for a multimodal drug delivery systems and early diagnosis of cancer cells [42, 43]. Owing to their large surface area, CNTs may be conjugated with a wide variety of ligands, such as proteins [44], peptides [45], enzymes [46], polysaccharides [47] and drugs [48]. These molecules can be bound to the CNT covalently or non-covalently (Fig. 1) [50]. These nano materials are hydrophobic in nature and thus insoluble in water, which muzzles their application in biomedical and medicinal chemistry [48]. Depending on the type of conjugated polymer, modified CNTs can be soluble in aqueous or organic solvents. In order to avoid the aggregation of CNTs, to render their hydrophilic characteristics and to facilitate their use for applications in drug delivery, biosensors, tissue engineering, cancer imaging, and thermal, various functionalization methods like adsorption, electrostatic interaction and covalent bonding are utilized with a number of compounds and 

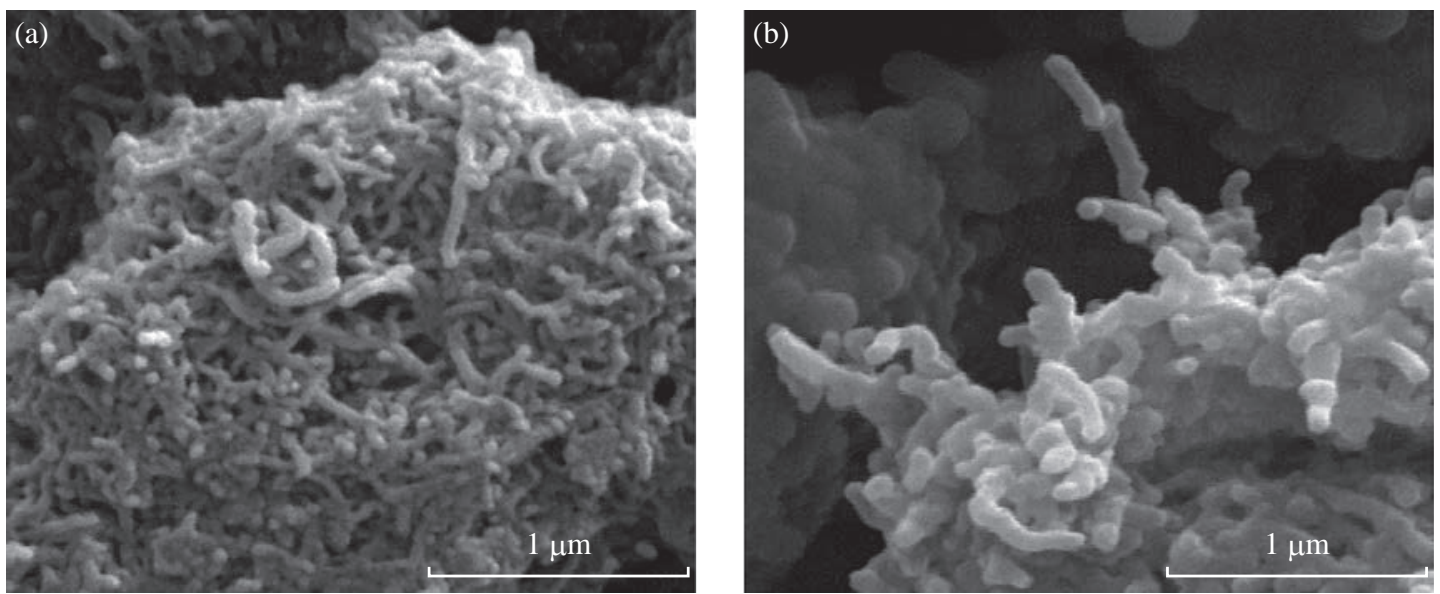

Fig. 1 (a) Scanning electron microscope images of SWCNT; (b) and modified SWCNTs with Taxol/Folic acid [50].

polymers [49, 50]. Compared with CNTs, graphene exhibits some important qualities such as low cost, facile fabrication and modification, the higher surface of area with two external surfaces and the absence of toxic metal particles which threaten the dominance of CNTs in many applications, including drug delivery, demonstrating lower toxicity and superior biocompatibility [51].

\section{Applications of CNTs in Cancer Therapy}

Currently, available technologies have made enormous advancement in cancer research, but an adequate therapy remains elusive [52]. Cancer is characterized as a disorder which arises following a number of mutagenesis steps, allowing cancerous cells to grow and divide uncontrollably. These processes allow cancer cells to acquire properties of unlimited proliferation potential, self-sufficiency in growth signals and resistance to both anti-proliferative and apoptotic cues which would, otherwise, contain their growth. Tumors have also evolved methods to gain further support through interactions with surrounding stromal cells, promoting their angiogenesis, their evasion of immune detection and their metastasis to distal organs [52, 53]. The Global Cancer Report issued by the World Health Organization (WHO) estimates that there are over 10 million new cases of cancer every year with over 6 million annual deaths caused by the disease [54]. The most frequent types of cancers causing death are, as listed by the WHO: lung, stomach, liver, colon and breast cancers [54]. Current cancer therapies can be broadly categorized into two groups: cytotoxic therapies and molecular targeted drugs. Examples of traditional cytotoxic therapies include radiation and chemotherapeutic compounds such as platinum-based drugs [55-57]. Radiation, utilizing photons, damages the DNA of cancer cells by creating free radicals, leading to the inhibition of cell division and, eventually, causing cell death. The current medical anticancer treatments involve applications where therapies are provided by removing the diseased cells (surgery), burning them out (radiation therapy), or poisoning the diseased cells faster than the healthy cells (chemotherapy), most of which do cause damage to healthy cells [5861]; moreover, present treatment methods are not very effective at stopping the spread or recurrence of cancer. One approach to overcome these drawbacks is design of nano-carriers which actively able to bind to specific cells after extravasations. This binding may be achieved by attaching targeting agents to the surface of the nano-carrier by a variety of conjugation methods. Then, nano-carriers will be recognized and bind in order to target cells through ligand-receptor interactions [62, 63]. A wide variety of ligands can potentially be employed to target cellular biomarkers including antibodies, [47] other proteins [47], peptides [45] and polysaccharides [46]. Improved targeting of carbon nanotubes (CNTs) to tumors is essential because larger tumors exhibit poor vascularization, particularly inside necrotic areas, resisting the localization of CNTs in the tumor. Additionally, no solvent is required for delivery of the drug, which means that undesired health effects from the solvent can be prevented; as well as, a lower concentration of the toxin is delivered to other parts of the body. Hence, the aftermath of chemotherapy descend striking by the patient undergoing treatment. Carbon nanotubes 
can also be used for hyperthermia treatment of cancer. The innate optical properties of CNTs, such as strong resonance Raman scattering and near infrared photoluminescence (NIR PL) in the 1.1-1.4 $\mu \mathrm{m}$ spectral region, make them useful biological imaging agents [64-68]. To image the nanoparticle uptake into tumors, through the enhanced permeability and retention effect (EPR) [69] is a key to nanomaterialbased cancer nanoparticles, such as nanotubes and gold nanoparticles, have been used as NIR contrast agents [70, 71]. Cancer cells are more susceptible to heat increases than healthy cells owing to the fact that the cancer cells are surrounded by blood vessels that have a strange flow result in dissipating heat less efficiently than blood around healthy cells [72]. However, to minimize the damage to healthy cells, heat still needs to be selectively delivered to tumor cells. The near infrared (NIR) (700 to $1100 \mathrm{~nm}$ ) is a special region where optical absorption in tissues is minimal and penetration is optimal and carbon nanotubes have a strong absorption in this region [73, 74]. Therefore using NIR light can effectively heat up CNTs in vivo. The high aspect ratios of CNTs are suitable for attaching some ligands such as folic acid with the aim to recognize folate receptors overexpressed on many tumors, including ovarian, breast, brain, kidney, lung, and liver [47]. Another multicomponent CNT-based system for cellular imaging applications in cancer therapy and magnetically guided drug delivery for local hyperthermia illustrate great undertaking in the harmless and effective transducer for cancer treatment. Magnetic nano-materials (MNP) based hyperthermia treatment has a number of merits compared to conventional hyperthermia treatment. In this method cancer cells absorb MNPs, thereby increasing the effectiveness of hyperthermia by delivering therapeutic heat directly to MNPs, can be targeted through cancerspecific binding agents, as a result of which it makes the treatment much more selective and effective. Thus, the rate of drug releasing can be controlled by directly modulating the magnetic field. In a nutshell, a number of types of magnetic nano-materials, ranging from well-known and well-investigated iron oxidebased nano-materials to metallic NPs such as Mn, $\mathrm{Fe}, \mathrm{Co}, \mathrm{Ni}, \mathrm{Zn}, \mathrm{Gd}, \mathrm{Mg}$, and their oxides have been investigated for their hyperthermic potential. Some of the hyperthermic agents based on iron oxide are magnetite NPs $\left(\mathrm{Fe}_{3} \mathrm{O}_{4}\right)$ stabilized by a variety of ligands such as dextran, [75-77] polyvinyl alcohol, hydro-gel and magnetic NPs $\left(\gamma-\mathrm{Fe}_{2} \mathrm{O}_{3}\right)[78,79]$, cationic liposomes, [80] stabilized with hydrophilic polymers such as starch or dextran and chitosan [81, 82]. Since the targeted therapy has the characteristics of driven magnetic accuracy, targeting, and high drugcapacity, it can be effective to lower toxic effects and to enhance the therapeutic effect $[43,44]$. Although, all these properties make this an ideal method for drug delivery, selectively targeted drug delivery systems are still facing challenges including improvement of specificity, stability, regulating bioavailability, and developing targeted carriers with lower toxicity [4765].

\section{Internalization of CNTs, Cellular Uptake of CNTs}

Many studies have confirmed the cellular absorption of CNTs. Nonetheless, it is however, not clear how CNT penetrates into cells. Successful chemotherapy, therapeutic agents should pass a series of biological barriers including hepatic and renal clearance, enzymolysis and hydrolysis, as well as cellular uptake and endosomal/lysosomal degradation [8385]. Various mechanisms for the internalization pathway of SWCNTs inside living cells have been proposed. Pantarotto et al. [84] suggested that insertion and diffusion of nanotubes across cell membranes is an energy-independent non-endocytotic process. In contrast, other reports have indicated that cell membrane transport of SWCNTs conjugates with proteins and DNA is through the Clathrin-receptor (mediated) dependent endocytosis pathway [86]. In a study conducted by Kam et al. [87], protein attached to SWCNTs-biotin was detected in the endosomes, suggesting that nanotubes uptaking occurred via endocytosis. It has been shown that MWCNTs and single-walled carbon nanotubes (SWCNTs) differ in their cell penetration mechanism. Additionally, the size of the CNTs influences their cellular uptake and fatality of cells, owing to the fact that long SWCNTs are localized in the cytoplasm, whereas short SWCNTs are transported into the nucleus, whereas the MWCNTs are excluded from the interior (Fig. 2) [88]. It has been proved that a proficient method is needed to minimize the noxious effects and also to increase the level of therapeutic response to CNTs as represented by their conjugation to a carrier molecule [89, 90]. For instance, various strategies for the fabrication of nano moieties to target folate receptors on the cancer cell membrane have been proposed [90]. Folate receptors are overexpressed in cancer and their targeting allows 

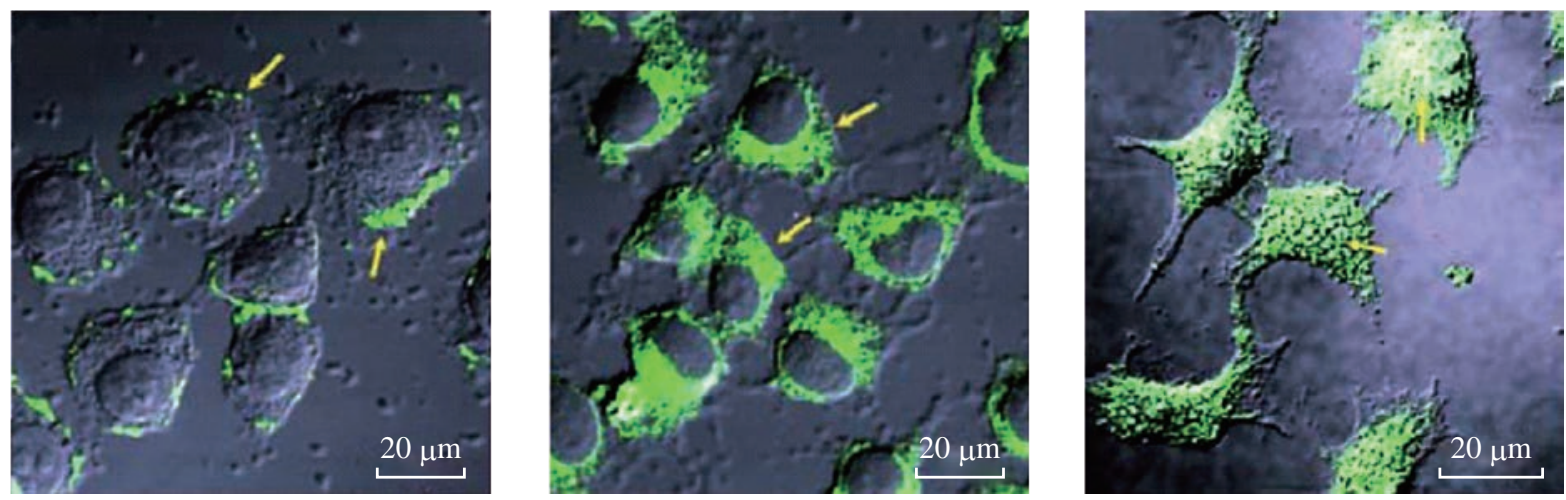

Fig. 2 Confocal microscopy images show that the ability of MWCNT, S-SWCNT and L-SWCNTs to be internalized into the cells, whereas the MWCNTs are excluded from the interior [88].

CNTs to facilitate cellular internalization of folate containing species by receptor-mediated endocytosis which is more selective than CNTs alone that enter cells through phagocytosis or endocytosis and through passive diffusion [91]. Most researchers have focused on the potential of CNTs for delivery of anticancer agents which might be attributed to their exclusive physicochemical features especially their ability for crossing various biological barriers within the body without generating an immunogenic response and toxic effects [92].

\section{Functionalization of CNTs}

Pristine, CNTs' inner and outer surfaces have different characteristics, offering the possibility of loading the inner space with biologically active species; moreover, modifying the outer surface to make the biocompatible nanotubes They tend to bundle up and are insoluble in most types of solvents [93] making it difficult to use them in biomedical and medicinal chemistry. In addition, some CNTs without any functionalization have been shown to be cytotoxic [9496]. Furthermore, bioactive agents can be conjugated to CNTs through functionalization, which as a result can serve as a drug carrier for drugs, antigens and gene delivery. Modification reactions of CNTs can be classified into two main approaches: (i) oxidation followed by carboxyl based couplings (ii) additional reactions to the sidewalls and tips of CNTs or noncovalent reactions.

In the first and most important approach, functionalization strategy consists of oxidation and carboxyl-based coupling. The carboxylic groups also allow covalent couplings with other molecules through amidation, esterification, thiolation, halogenation, hydrogenation, and cycloaddition reactions as well as radical, carbene and electrophilic additions [44].

The second strategy is through additional reactions employed to attach some organic groups to the sidewalls and/or tips of the CNTs. Strategies for noncovalent modification include the use of: (a) organic solvents [97], (b) organic polymers [98, 99], (c) amphiphilic peptides, (d) nucleic acids [100], sugars, and (e) detergents [101].

One of the largest applications for drug delivery is in cancer treatment, where proper amounts of drugs need to be directed to the targeted tissue while minimizing unwanted effects of the drugs on normal tissues. Several water-soluble polymers have been covalently and non-covalently attached onto the surface of CNTs to produce modified water-soluble CNT-polymer (Table 1). Depending on the type of conjugated polymer, modified CNTs can be soluble in aqueous or organic solvents, for example, conjugation of polylactide or polycaprolactone onto the surface of CNTs tends to solubilization of CNTs in organic solvents such as chloroform [102, 103]. It has been proved that polymers not only raise the functionality, biocompatibility and water solubility of CNT but also are able to change the CNTs conformations dramatically [102-104]. According to CNTs' polymeric and cationic innate abilities, soluble functionalized carbon nanotubes seem to represent an innovative drug delivery system, since they are capable to penetrate into the cell without altering their morphology [105, 106]. It is well worth noting that functionalized carbon nanotubes with Fluorescein isothiocyanate (FITC) or a fluorescent bioactive peptide peptide are able to cross the cell membrane, while the CNT with the FITC mainly distributed into the cytoplasm and slowly moved into the nucleus, the CNT with the fluorescent 
Table 1 Carbon nanotube based targeted delivery system

\begin{tabular}{|c|c|c|c|c|}
\hline Compound & Drug & Targeting agent & Tumor & Ref. \\
\hline SWCNT-PTX-FA-FITC & PTX & FA, FITC & Breast cancer; Cervical cancer & 50 \\
\hline MWCNT-MTX & MTX & FITC & - & 108 \\
\hline SWCNT-PTX-PEG & PTX & - & Breast cancer & 109 \\
\hline SWCNT-PL-DOX & DOX & RGD & Breast cancer & 110 \\
\hline SWCNT-CHI-DOX & DOX & FA & Liver cancer & 111 \\
\hline SWCNT-ALG-CHI-DOX & DOX & FA & Cervical cancer & 112 \\
\hline SWCNT-PEG-DOX & DOX & FA & Breast cancer & 113 \\
\hline MWCNT-PEG-PTX & PTX & FA, QD & - & 114 \\
\hline SWCNT-PEG-Pt (IV) & cisplatine & FA & - & 55 \\
\hline MWCNT-PCA-PEG-PCA & cisplatine & - & Colon adenocarcinoma & 115 \\
\hline MWCNT-PCA-PTX & PTX & - & - & 116 \\
\hline SWCNT-FITC-Biotin & Taxoid & FITC & Leukemia & 117 \\
\hline SWCNT-BSA-FITC-DOX & DOX & FITC & Colon cancer & 118 \\
\hline
\end{tabular}

peptide rapidly penetrated into the nucleus. These CNT conjugates entered the cell with a passive mechanism. In fact, the translocation capacity was not modified by decreasing the temperature or by using inhibitors of endocytosis-mediated process. One strategy to specifically deliver drugs to a tumor site is through the enhanced permeability and EPR [34]. The EPR effect is due to leaky vascular structures and an impaired drainage system of tumors. Therefore, nanoparticles with sizes ranging from 100 to $700 \mathrm{~nm}$ cannot penetrate the pores of the blood vessels in healthy tissue but will go through the pores of tumor blood vessels and preferably accumulate at the tumor site $[107,108]$. CNTs can be conjugated with a therapeutic agent and will preferably accumulate at the tumor through the retention effect [109-118].

\section{Non-covalent method}

Non-covalent attachments have received much interest owing to the possibility of linking different groups of polymers on the surface of CNTs without disturbing their bonding network [119]. One of the main problems in using CNTs as drug nanocarriers is their recognition as foreign particles in the bloodstream. Non-covalent adjustment approaches of CNTs have mainly been set up on the three general path ways comprising hydrophobic connections between the surface of CNTs and long-chain hydrophobic polymers, $\pi-\pi$ interactions between CNTs and polymers containing a sector with the ability of producing " $\pi-\pi$ stacking" interactions such as pyrene, ferrocene and other aromatic containing molecules [119, 120], finally electrostatic and hydrogen-bond interactions between CNTs or acid-treated CNTs (-COOH) and macromolecules or polymers with polar functional groups which is named "polymer wrapping" mechanism [121]. Polyethylene glycol (PEG) is one of the most popular CNT surface modifiers, due to its hydrophilicity, flexibility and biocompatibility [122]. PEG-modified CNTs may also be dispersed in relatively higher salt-concentrated aqueous solutions [123]. The optimized CNT-PEG conjugate afforded relatively low reticuloendotheelial system (RES) accumulation, high tumor uptake and low skin retention, thus making CNT an ideal choice for in vivo cancer treatment [124]. The cellular internalization of the CNTs depends, among other factors, on the PEGchain length; the longer PEG chains reduce cellular uptake [125].

In such an assay, large surface areas exist for supra-molecular chemistry on single-walled carbon nanotubes pre-functionalized non-covalently or covalently by common surfactant or acid-oxidation routes. Water-soluble CNTs with PEG-functionalization via these routes allow surprisingly high degrees of $\pi-\pi$ stacking of aromatic molecules, including a cancer drug with ultrahigh loading capacity, a widely used fluorescence molecule (fluorescein), 
and combinations of molecules. Liu et al. [109] have shown that there is much space for supra-molecular chemistry assembly of molecules on SWCNTs pre functionalized non-covalently by common surfactant or acidoxidation routes. Aqueous soluble SWCNTs with PEG functionalization by these routes allow for $\pi-\pi$ stacking of various aromatic molecules, including doxorubicin (DOX) as a cancer chemotherapy drug (DOX) with an ultrahigh loading capacity of _400\% by weight and a widely used fluorescence molecule (fluorescein) [126]. Biologically inert SWCNTs with non-covalent PEG functionalization (PL-PEG5400NH) have been conjugated to antibodies such as Rituxan to selectively recognize CD20 cell surface receptor on B-cells with little nonspecific binding to negative T-cells and Herceptin to recognize HER2/neu positive breast cancer cells [109]. These results have suggested that unoccupied surface areas on functionalized SWCNTs were useful for binding of other molecules. It is suggested that non-covalent binding of DOX on SWCNTs most likely occurred via $\pi$ - $\pi$ stacking [127] and hydrophobic interactions owing to the aromatic nature of the DOX molecule and the relatively low solubility of deprotonated DOX at basic conditions. Free, unbound DOX exhibited high fluorescence, while weak fluorescence was observed for DOX after binding to SWCNTs. This high degree of fluorescence quenching is evidence of $\pi-\pi$ stacked DOX, similar to other aromatic molecules $\pi-\pi$ stacked onto nanotubes [126-128]. This represents a novel partition and utilization of the SWCNT sidewall surface area affording a unique and interesting way to attach drugs to carbon nanotubes in view of their extended polyaromatic sidewalls. In another investigation to demonstrate the targeted delivery of doxorubicin by SWCNTs, they conjugated a cyclic RGD peptide on the terminal groups of PEG on SWCNTs, imparting a recognition moiety for integrin receptors upregulated in a wide range of solid tumors. RGDlabeled PL-PEG5000 $\mathrm{NH}_{2}$ has been covalently used for loading of anticancer drug, doxorubicin, on the surface of SWCNTs to kill glioblastoma and breast cancer cells. The loading interactions between DOX molecules and SWCNTs surface is strong enough to prevent rapid desorption in normal physiological conditions. Molecular release of the non-covalently bound molecules can be triggered by environmental changes such as PH or other external stimuli. This system has also been used for loading/releasing other aromatic molecules such as fluorescent dye molecules
Fluorescein isothiocyanate and another aromatic chemotherapy agent (Doxorubicin) [129].

Another frequently-studied non-covalent interaction is a targeted drug delivery system based on polysaccharide [sodium alginate (ALG) and chitosan (CHI)] modified carboxylate SWCNTs for controlled release of DOX; in addition, including folic acid as a targeting agent [130]. The CHI/ALG-SWCNTs were initially oxidized and cut and then the appropriate polysaccharide (ALG or CHI), leading to the encapsulation of the SWCNTs by the polysaccharides [129-132]. According to the polymer wrapping process involving initial treatment with ALG followed by CHI, the SWCNT core could be doubly wrapped by both CHI and ALG. Afterwards, CHI/ALG-SWCNTs with DOX in aqueous solution also allows the DOX to be attached to the nanotubes non-covalently [133]; moreover, chitosan reacts more efficiently with FA via an amidation reaction offering a further advantage over the polymer coated nanotubes in that one polymer has an affinity for the DOX drug and one for the FA targeting molecule. Consequently, the amount of DOX loading was monitored by ultraviolet-visible (UV-Vis) absorption spectroscopy [121]. According to the zeta potentials data of the modified SWCNT the positively charged DOX molecules are more readily absorbed onto the surfaces with lower surface potentials, suggesting that electrostatic interactions as well as $\pi-\pi$ stacking interactions play an important role with respect to DOX loading [121]. Cell viability test results indicate that incubations with the unloaded nanotubes or the free drug result in appreciable cytotoxicity at the administered concentrations, indicating that the DOXFA-CHI/ALG-SWCNTs are not only cytotoxic, but also selective. It is suggested that the DOX has been released in the low $\mathrm{PH}$ environment of the lysosomes, then migrating into nucleus to bind DNA. This latter interaction has been revealed to inhibit transcription and ultimately leads to cell death [133-135].

\section{Covalent method}

The use of carbon nanotube covalent functionalization to deliver anticancer agents has also been recently explored. The functionalization of carbon nanotubes for biomedical applications involves covalent modifications. Covalent modifications are based upon linking of already functionalized or commercially available polymer chains onto the surface of CNTs by suitable chemical reactions [136]. In biological 
applications, oxidation, amidation, esterification, radical coupling, and grafting polymers on the sidewalls of carbon nanotubes are widely adopted. However, in the grafting approach, first appropriate functional groups are created on the surface of CNTs, and then, desired polymers will be grown on the nanotube surface via polymerization reactions [136].

One of the recently reported example of this category is about effective pointing of an anticancer is conjugated methotrexate (MTX) by modified MWCNT. It has been suggested that the covalent functionalization of MWCNTs with MTX was achieved via a 1,3-dipolar cycloaddition approach [137]. In this survey, a methodology was developed for the introduction of two linking sites on the sidewalls of carbon nanotubes for FITC and MTX sequentially. However, the drug releases profiles in vitro and in vivo efficacy were not evaluated. Antitumor agent 10 hydroxycamptothecin (HCPT) was conveyed by a covalently modified MWCNT [128]. Diaminotriethylene glycol was used as a spacer linking the carboxylic groups on the oxidized nanotube to drug molecules via amidation reaction. The MWCNT formulated HCPT showed an improved antitumor activity both in vitro and in vivo compared to the clinical HCPT formulation and the MWCNTHCPT conjugates have a longer blood circulation and a higher drug accumulation at the tumor site [137].

In a different approach, chemically functionalized SWCNTs have shown promise in tumor targeted accumulation in mice and exhibit biocompatibility, excretion and little toxicity. On this base, SWCNTs were first functionalized with branched poly (ethylene glycol) chains; afterwards, PTX was linked to the branched of PEG via amide bonds [119]. The extent research reveals that conjugated paclitaxel (PTX), a widely used cancer chemotherapy drug to branched polyethylene-glycol chains on SWCNTs via a cleavable ester bond to obtain a water soluble SWCNT-paclitaxel conjugate (SWCNT-PTX). SWCNT-PTX affords higher efficacy in stifling tumor growth than clinical paclitaxel in a murine 4T1 breast-cancer model, owing to prolonged blood circulation and 10-fold higher tumor PTX uptake by SWCNT delivery likely through enhanced permeability and retention effect. The SWCNT-PTX complexes were shown to stay in the blood for longer times (81.4 $\pm 7.4 \mathrm{~min})$ than drug (18.8 $\pm 1.5 \mathrm{~min})$ and PTX coated with PEG (22.8 \pm 1.0 min). This prolonged circulation time together with EPR effects allowed for much higher accumulation of the drug at tumor sites (10-fold higher than PTX and 6-fold higher than PEG-PTX after $2 \mathrm{~h}$ of injection and 6- and 4-fold higher, respectively, after $24 \mathrm{~h}$ of injection) [109]. In conclusion, the high delivery efficacy of PTX by SWCNT subsequently resulted in tumor inhibition of SWCNT-PTX (5 mg/kg PTX) for the 4T1 tumor model known to be resistant to PTX treatments [138-143]. It has been shown that nanotube drug delivery is promising for high treatment efficacy and minimum side effects for future cancer therapy with low drug doses.

In another study, SWCNTs have been a prefunctionalized covalently with PTX-succinic anhydride-modified, and folic acid, as a targeting agent for many tumors toward cancer cells in order to open up new opportunities in chemical, biological, and medical applications of novel nano-materials (Fig. 3) [50]. In this approach both the FA and PTX covalently functionalized upon amide- SWCNTs were well-solubilized and stable in water, PBS, and cell medium containing $10 \%$ fetal calf serum and full serum and biocompatibility and high targeting ability. Atomic force microscopy was used to measure the diameter and length of the functionalized SWCNTs after deposition on a Si substrate. Moreover, in order to insert the covalently conjugated onto to modified SWCNTs prepared by 1-(3-(dimethylamino)

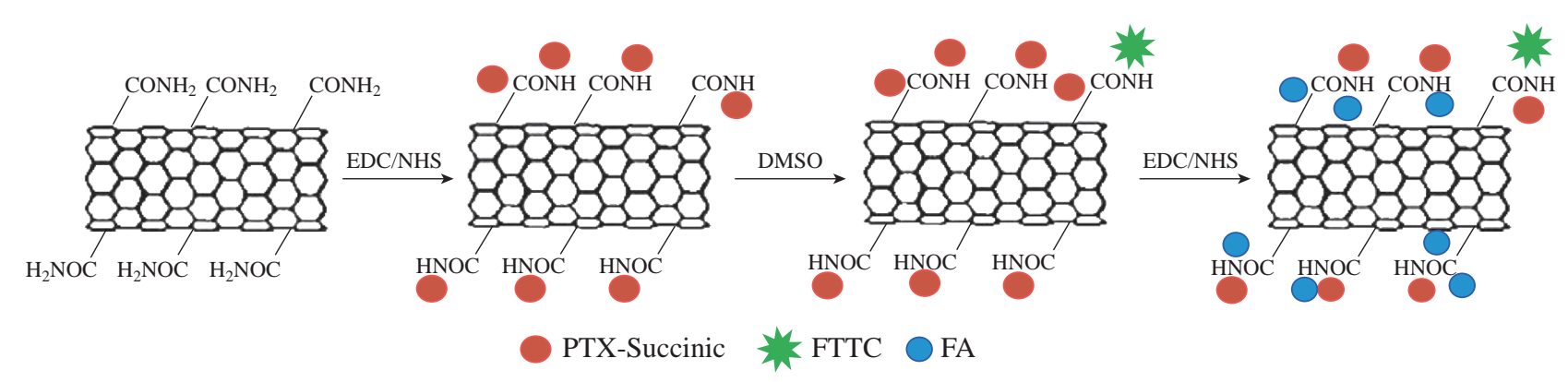

Fig. 3 The preparation of SWCNT with targeting properties involves several steps: coating SCNTs with paclitaxel, attaching Fluorescein isothiocyanate probe to the SWCNT-PTX, and finally targeting molecules (FA) attachment [50]. 
propyl)-3-ethylcarbodiimide hydrochloride (EDC) and N-hydroxysuccinimide (NHS) [144, 145]. It is suggested that the drug was successfully loaded onto the SWCNTs, which may be mainly broken up by covalent stacking and hydrophobic interactions. The concentration of PTX loaded onto SWCNTs was measured by the absorbance peak at $240 \mathrm{~nm}$ with a molar extinction coefficient of $31.7 \times 10^{5} \mathrm{M} \mathrm{cm}^{-1}$. Loaded onto the nanotubes the quantity of PTX was measured by UV-Vis-NIR spectra, for the same batches of samples [50].

Since the application of PTX in clinical therapy is limited by poor aqueous solubility, inefficient distribution, and the lack of selectivity, modification of PTX with succinic acid (the cleavable linker) CNTs exhibited good aqueous solubility, biocompatibility [144]. According to the latest results polymers change the physicochemical property of CNTs in biological medium and ability of polymers to develop the efficacy of CNTs as drug delivery systems. It is the consequence of secondary interactions among these two portions. The superior mechanic strength and chemical inert also makes it ideal for blood compatible applications, Moreover the functionalized CNTs have been extensively explored for in vivo targeted drug, which will potentially improve the efficiency of many cancer treatments (Fig. 4) [50].

\section{Carbon Nanotubes-Magnetic Nanoparticles Assemblies}

Over the past few years, researchers have developed various types of nanoparticles for treatment of cancer [146-151]. Magnetic nanoparticles have received pervasive attention in medicine communities owing to their potentials in medical applications [146-151]. These applications associate closely with the unique magnetic properties of MNPs with comparable size to biologically important objects. Magnetic CNTs have also illustrated favorable outcomes as a MRI contrast agent with high nuclear magnetic resonance relaxivities, little cytotoxicity, catalysis [152], magnetic

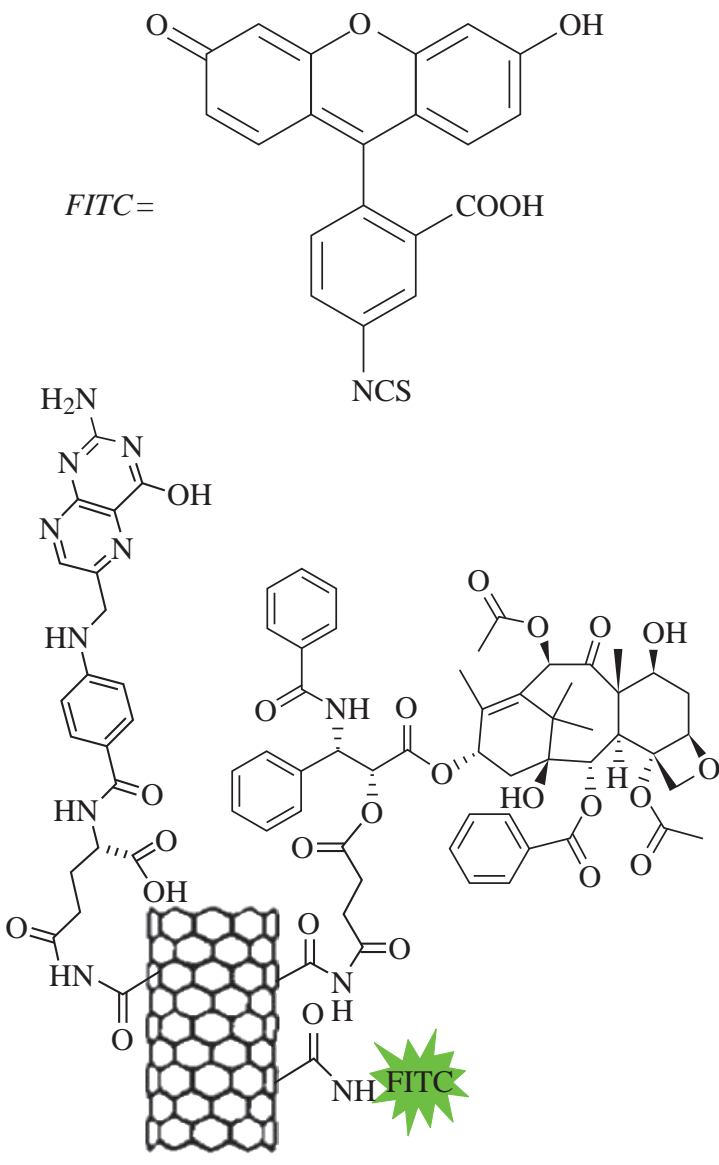

(a)
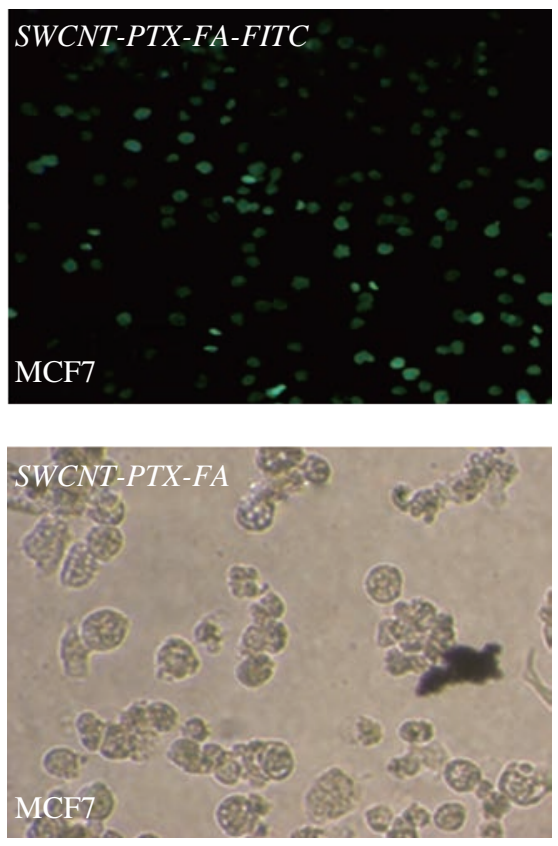

(b)

Fig. 4 (a) Chemical formula of the SWCNT/PTX-FA/FITC (SWCNTs functionalized with paclitaxel and folic acid at on the sidewall by covalent bond); (b) confocal fluorescence images of MCF-7 breast cancer cells treated with SWCNT/PTX/FA [50]. 
hyperthermia [153] and high cell-labeling efficiency [154]. As mentioned earlier, CNTs possess also a hollow cavity that can be filled with a variety of metals such as $\mathrm{Au}, \mathrm{Ag}, \mathrm{Cu}, \mathrm{Sn}, \mathrm{Fe}, \mathrm{Co}$, and $\mathrm{Ni}$ and employed as nano-antennas or microscopic probes [154-156]. These main reasons evolve in many studying in order to functionalize (or coating) of CNTs such as magnetic or super paramagnetic nanoparticles or in filling their cavity with magnetic molecules in order to obtain versatile systems being able to be employed more efficiently in biomedical or bio-imaging applications. In fact, the biological molecular could be tagged on the MNPs, which are monitored and manipulated in the presence of external magnetic fields [157-160]. The chemical combination of magnetic nanoparticles or nano-crystals and CNTs in order to obtain nanohybrid structures, follows various strategies including, encapsulation of magnetic molecules inside the carbon nanotubes or grafting/decorating CNTs on their surface by bio-conjugation chemistry or electrochemical deposition. The strategies to attach some ready synthesized nano-crystals have been achieved using covalent bonds [159], electrostatic interactions [158], $\pi-\pi$ stacking [159], and hydrophobic interactions [160]. Many other strategies have been devised in the last few years and we will discuss separately their applications, according to their various application.

\section{Covalent method}

One of the covalent attachment strategies [161] has received much interest owing to the possibility of grafting gold nano-rods and multi walled carbon nanotubes and developed a simple and effective strategy to prepare NIR absorption enhancer MWCNTs through covalent interaction of carboxyl groups on the MWCNTs with silica-coated gold nano-rods (sGNRs). Then, modified Gold nanorods peptides with the amino silane agent were conjugated with the sGNRMWCNT hybrid structure in a struggle to eliminate their cytotoxicity and improving their biocompatibility, as a result of which RGD-conjugated sGNR-MWNT (RGD-GNR-MWNT) nano-probes were used for photoacoustic imaging of in vivo gastric MGC803 and GES-1 cells. Gold nano-rods, due to their small size, strong light-enhanced absorption in the NIR, and plasmon resonance-enhanced properties have become attractive noble nano-materials for their potential in applications including, photothermal therapy [162], bio-sensing [163], PA imaging [164], and gene delivery [165] for cancer treatment. According to the cell viability data RGD-GNR-MWNT nanoprobes revealed good biocompatibility to MGC803 and GES-1 cells within the dose of $800 \mu \mathrm{g} / \mathrm{mL}$ in the medium. These results illustrated that RGD-conjugated sGNRs/MWNTs may be a good contrast agent for photoacoustic imaging of in vivo gastric cancer cells, and gold nano-rods can enhance the photoacoustic (PA) signal of MWNTs. Golden single-walled carbon nanotubes have been used for photoacoustic imaging of in vivo tumors [166, 167]. Additionally, regarding that RGD-conjugated sGNR/MWNT probes exhibited good water solubility and low cellular toxicity, it could target in vivo gastric cancer cells, and obtained strong photoacoustic imaging in the nude model.

In a recent work, the application in cancer targeted imaging and magnetically guided drug delivery was reported by Yang et al. [75], where a novel magnetic lymphatic-targeting drug-delivery system, based on functionalized carbon nanotubes, was presented in order to improve cancertherapy with lymph node involvement. Multiwalled carbon nanotubes have been covalently functionalized with magnetic poly-acrylic acid (PAA) in order to obtain MWCNTs-GEM and deliver the targeted cell of this novel lymphatic (Fig. 5(a)). Gemcitabinis (GEM) a nucleoside analogue and a S-phase specific cytotoxic agent shown activity in many solid tumors, such as pancreatic cancer, nonsmall cell lung cancer, head and neck squamous cell cancer, germ cell tumors, and tumors of the bladder, breast, ovary, cervix and biliary tract, as well as some hematologic malignancies [76]. It is suggested that targeting drug delivery system using functionalized multiwalled carbon nanotubes can be effective for cancer treatment. The mMWCNTs/GEM was subcutaneously injected into a mouse that had cancer lymph node metastasis via the left rear footpad, and were taken up into lymphatic vessels and retained in the targeted lymph node under the magnetic field (Fig. 5(b)). The in vitro cell cytotoxicity after various treatments was assessed at given time functionalized PAA-MWCNTs designed with magnetite nanoparticles $\left(\mathrm{Fe}_{3} \mathrm{O}_{4}\right)$ can be efficiently taken up by lymphatic vessels and delivered to regional lymph nodes in vivo with little toxicities. In addition, this magnetic lymphatic targeting system [77] can deliver gemcitabine into the lymph nodes in vivo with high efficiency under the guidance of magnetic field [168].

\section{Non-covalent method}

Recently, groundbreaking efforts have been made 


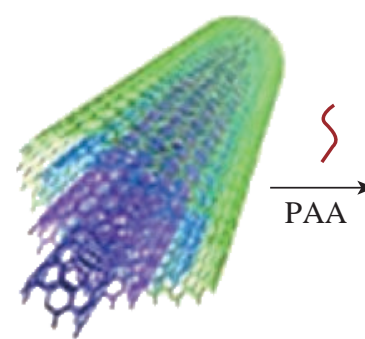

MWNTs

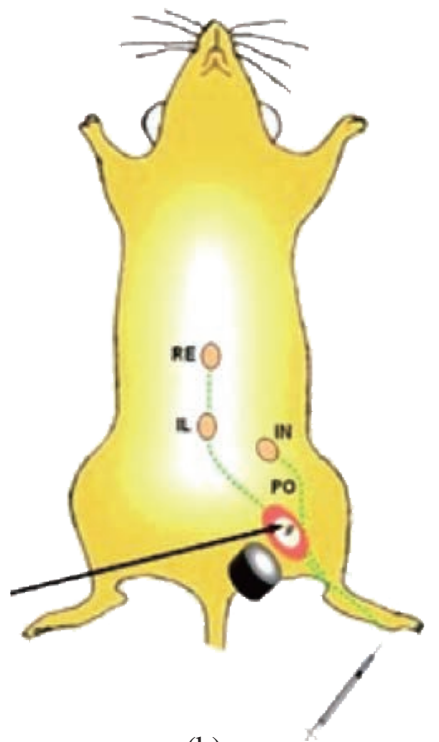

(b)

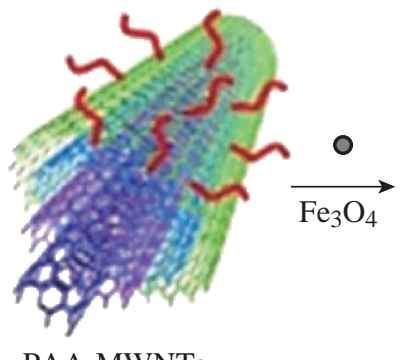

PAA-MWNTS

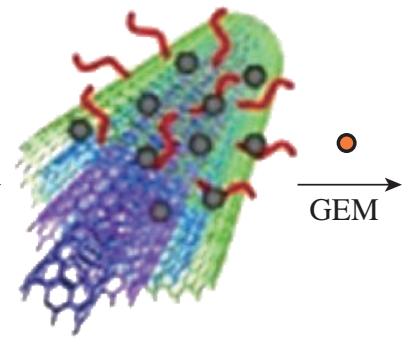

PAA-mMWNTs

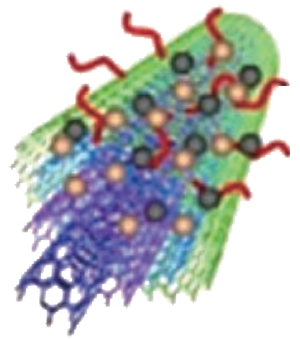

PAA-mMWNTs-GEM

(a)

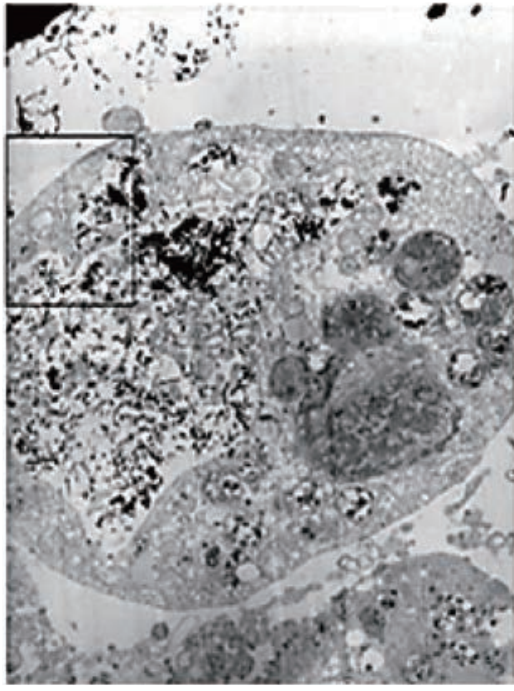

(c)

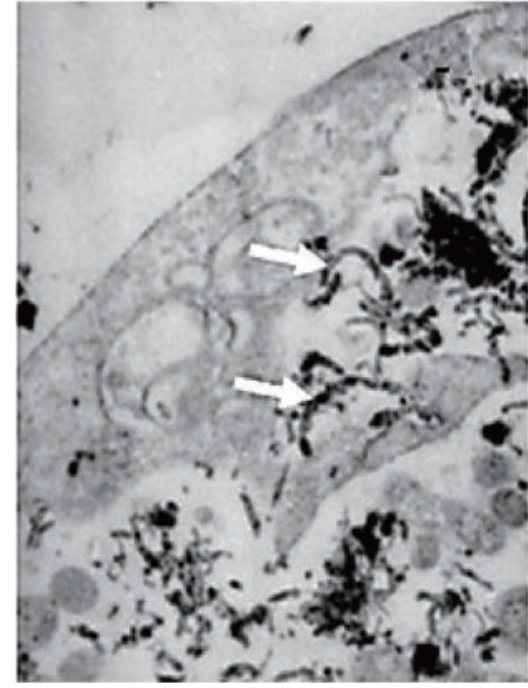

(d)

Fig. 5 The preparation of the Magnetic lymphatic drug delivery system, (a) magnetic lymphatic targeted chemotherapy in mice and illustration of chemical reactions used to attach gemcitabine onto mMWCNTs; (b) magnetic lymphatic targeted chemotherapy in mice. MMWCNTs/GEM was subcutaneously injected into a mouse that had cancer lymph node metastasis and TEM image (c) and (d) of mMWCNTs-treated human pancreatic cancer cell line. The white arrows point to mMWCNTs inside the cytoplasm [75].

for fabricating magnetic carbon nanostructures by conjugating MNPs with carbon nanotubes (CNTs) [169]. However, the attachment of iron oxide nanoparticles and QDs on the active surface of CNTs considerably plummeted the CNTs capacity for drug storage and further functionalization [170, 171]. Additionally, direct contact of Quantum dots with iron oxide nanoparticles on CNTs surface causes fluorescence quenching and diminishes the magneticfluorescent dual functionality. An effective method to incorporate CdTe quantum dots with $\mathrm{Fe}_{2} \mathrm{O}_{3}$-filled carbon nanotubes (CNTs) with different capping agents onto the surface of functionalized carbon nanotube has been developed for the first time [64]. In this work, $\mathrm{Fe}_{2} \mathrm{O}_{3}$ is filled the hallow cavity of CNTs facilitating magnetically guided delivery and improves the synergetic targeting effciency [64]. MNPs were encapsulated into the interior cavity of CNTs with the aim of diminishing the magnetic-fluorescent dual functionality and minimize the potential toxic side- effects by exposure of free magnetic nanoparticle to biological environment $[64,65]$. Moreover, the biocompatible and chemically inactive hybrid $\mathrm{SiO}_{4}$ shells provide a cover layer to mitigate the toxicity of Quantum dots and to protect its fluorescence from being quenched by CNTs; afterwards, poly (sodium 4-styrenesulfonate) (PSS) was absorbed onto the surface of modified magnetic CNTs via $\pi-\pi$ interactions, afterwards non-covalent interactions between poly allylamine (PHA)-Hybrid $\mathrm{SiO}_{2}$-coated QDs (HQDs) nanoparticles and PSS-coated $\mathrm{Fe}_{2} \mathrm{O}_{3} / C N T$ have resulted in preparation of $\mathrm{Fe}_{3} \mathrm{O}_{4} / \mathrm{CNT}$-HQDs. The CdTe nanoparticles were coated with a thin $\mathrm{SiO}_{2}$ layer containing $\mathrm{Cd}^{2+}$ and thioglycolic acid (TGA). $\mathrm{Fe}_{3} \mathrm{O}_{4} /$ CNT-HQDs-Trf forms through the interaction between formed amine-reactive Trf-NHS ester and $\mathrm{Fe}_{2} \mathrm{O}_{3}$ /CNTHQDs by amide bond. Eventually, doxorubicin loaded onto $\mathrm{Fe}_{3} \mathrm{O}_{4} / \mathrm{CNT}$-HQDs-Trf via $\pi-\pi$ interactions. The efficacy of drug delivery systems for killing the cancer cells and targeting the drugs towards tumors has been 
investigated. Interestingly, in this work, doxorubicin released from $\mathrm{Fe}_{3} \mathrm{O}_{4} / \mathrm{CNT}$-HQDs-Trf is highly $\mathrm{pH}$ dependent, and a very limited amount of DOX is released at $\mathrm{pH} 8.5$ and 7.4. It is of vital importance that the selective delivery of CNTs to tumor cells- through the interaction between transferrin and receptors of the Hela cells- minimizing their side effects can be achieved by simultaneously anchoring iron oxide nanoparticles and $\mathrm{Fe}_{3} \mathrm{O}_{4}$ /CNTHQDs-transferrin followed by using an external magnetic field. It is noticeable that the uptake of $\mathrm{Fe}_{3} \mathrm{O}_{4} / \mathrm{CNT}$-HQDs-Trf is further remarkably improved, for in the presence of an external magnetic field $[172,173]$.

\section{Toxicity of Carbon Nanotubes}

It is of vital importance to determine carbon nanotubes' pharmacological and toxicological profiles in order to successfully apply a new generation of nano-materials as nano-medicine [173]. Successful interpretation of these results is difficult because the CNTs can significantly disrupt the emission of the certain particles [174-178]. Although the molecular and cellular mechanisms for the cytotoxicity and the entering of CNTs into cells have not been completely understood, functionalized carbon nanotubes are successfully able to cross cell membranes via an endocytosis pathway or endocytosis independent mechanism. Toxicity and pathology engendered by CNTs on an organismal in both in vivo and in vitro studies have been attributed to various factors in order to determine the nature of nano-toxicity in living organisms and cells including number of walls, length and aspect ratio, surface area, degree of aggregation, rigidity, extent of oxidation or functionalization, purity, hydrophobicity, surface topology, method of administration, dispersibility, type and degree of functionalization, and method of manufacture (which can leave catalyst residues and produce impurities) [28]. Additionally, toxicity of CNTs is also dependent on their concentration, dose, duration and method that cells or organisms are exposed to and even the utilized dispersant to solubilize the nanotubes. The length and shape of CNTs influence how well they cross the membrane of macrophages and determine the resulting immunologic response [64, 172]. For example, following synthesis, residual metal (Co, Fe, $\mathrm{Ni}$ and $\mathrm{Mo}$ ) is normally encapsulated in a layer of carbon, either amorphous soot or layers of graphite [179]. It is strongly suggested that one way of doing so is toss ultra-sonication owing to the fact that ultrasonication promotes the release of a metallic impurities into solution [180]. It has been discovered that metal impurities is one of the main factors that determines CNTs toxicity, resulting in cell death through various mechanisms such as mitochondrial destruction and oxidative stress [181].

To that end, scientists have examined CNT toxicity in several cell lines [182, 183], as well as using in vitro studies to divulge the effect of various factors on CNT toxicity, in vivo studies have also played a major role for investigating the CNT toxicity. Regardless of the drawbacks of in vitro studies, they have been invaluable in identifying important side effects of CNTs. In vivo studies are favored over in vitro studies, as long as they allow nanotoxicity to be measured on an entire organism, rather than in cell culture. Compared to pristine CNTs, well-functionalized CNTs by biocompatible coatings such as PEGylation exhibit remarkable reduction of toxicity in vivo after being intravenously injected into animals [82, 184-188].

Nonetheless, the extent research reveals that as well as others, specific types of functionalization CNTs can greatly minimize CNT toxicity and represent promising progress towards their pharmacological applications.

\section{Conclusions}

In summary, this study discusses some chemical methods to modify CNTs for the drug delivery. An ideal CNT is expected to enter and destroy cancer cells while minimizing the accumulation and side effects to normal organs. CNTs appear to accumulate in cancerous tumor tissue much more than in normal tissues, partly due to the EPR effect. The reported data indicate a potential of modified CNTs in different biomedical applications, particularly as a valuable tool in anticancer drug delivery due to their good aqueous solubility and biocompatibility. It is obvious that polymers change the conformation and shape of CNTs from strand-type toward closed conformations. Finally, investigations on all possible ways for interactions of CNTs and polymers lead to better knowledge in the design of novel anticancer drug delivery systems.

\section{Competing Interests}

The authors declare that they have no competing interests. 


\section{References}

[1] E. Biazar, S.K. Heidari, The healing effect of stem cells loaded in nanofibrous scaffolds on full thickness skin defects. J. Biomed Nanotechnol, 2013, 9: 1471-1482.

[2] M. Rezaei-Tavirani, E. Biazar, J. Ai, et al., Fabrication of collagen-coated poly (beta-hydroxy butyrate-cobetahydroxyvalerate) nanofiber by chemical and physical methods. Orient. J. Chem, 2011, 27: 385-395.

[3] J. Ai, S.K. Heidari, F. Ghorbani, et al., Fabrication of coated-collagen electrospun PHBV nanofiber film by plasma method; and its cellular study. J. Nanomater, 2011, 2011: 1-8.

[4] E. Biazar, S.K. Heidari, Chitosan-cross-linked nanofibrous PHBV nerve guide for rat for sciatic nerve regeneration across a defect bridge. ASAIO J, 2013, 59: 651-659.

[5] M. Sahebalzamani, E. Biazar, M. Shahrezaei, et al., Surface modification of PHBV nanofibrous mat by laminin protein and its cellular study. Int. J. Polymer Mater Po, 2015, 64: 149-154.

[6] E. Biazar, S.K. Heidari, Design of oriented porous PHBV scaffold as a neural guide. Int. J. Polymer Mater Po, 2014, 63: 753-757.

[7] E. Biazar, S.K. Heidari, Gelatin-modified nanofibrous PHBV tube as artificial nerve graft for rat sciatic nerve regeneration. Int. J. Polym Mater Po, 2014, 63: 330-336.

[8] R. Zeinali, E. Biazar, S.K. Heidari, et al., Regeneration of full-thickness skin defects using umbilical cord blood stem cells loaded into modified porous scaffolds. ASAIO J, 2014, 60: 106-114.

[9] E. Biazar, R. Zeinali and N. Montazeri, Regeneration of full-thickness skin defects using umbilical cord blood stem cells loaded into modified porous scaffolds. ASAIO J, 2014, 60: 106-114.

[10] A. Sahebalzamani, E. Biazar, Modification of poly caprolactone nanofibrous mat by laminin protein and its cellular study. J Biomater Tiss Eng, 2014, 4: 423-429.

[11] H. Tahermansouri, E. Biazar, Functionalization of carboxylated multi-wall carbon nanotubes with 3,5-diphenyl pyrazole and an investigation of their toxicity. New Carbon Mater, 2013, 28: 199-207.

[12] Z. Roveimiab, A.R. Mahdavian, E. Biazar, et al., Preparation of magnetic chitosan nanocomposite particles and their susceptibility for cellular separation applications. Journal of Colloid Science and Biotechnology, 2012, 1: 82-88.

[13] H. Tahermansouri, E. Biazar, Functionalization of carboxylated multi-wall carbon nanotubes with 3, 5-diphenyl pyrazole and an investigation of their toxicity. Carbon, 2013, 63: 594.

[14] J. Azizian, H. Tahermansouri, E. Biazar, et al., Functionalization of carboxylated multiwall nanotubes with imidazole derivatives and their toxicity investigations. Int. J. Nanomed 2010, 5: 907-914

[15] F. Ghasemvand, E. Biazar, S. Tavakolifard, et al., Synthesis and valuation of multi-wall carbon nanotubepaclitaxel complexas an anti-cancer agent. International journal of polymeric materials, 2014, 63: 898-908.

[16] E Biazar, S.K. Heidari, M.T. Rezaei, et al., Bone formation in calvarial defects by injectable nanoparticular scaffold loaded with stem cells. Expert Opin. Biol Ther, 2013, 13: 12-1653.

[17] A. Baradaran-Rafii, E. Biazar, S.K. Heidari, et al., Oriented nanofibrous silk as a natural scaffold for ocular epithelial regeneration. J Biomat Sci-Polym E, 2015, 26: $1139-1151$.

[18] A. Baradaran-Rafii, E. Biazar and S. Heidari-keshel, Cellular response of limbal stem cells on PHBV/Gelatin nanofibrous scaffold for ocular epithelial regeneration.
Int. J. Polym Mater Po, 2015, 64: 879-887.

[19] A. Baradaran-Rafii, E. Biazar and S. Heidari-keshel, Cellular response of stem cells on nanofibrous scaffold for ocular surface bioengineering. ASAIO J, 2015, 61: 605612.

[20] A. Baradaran-Rafii, E. Biazar and S. Heidari-keshel, Cellular response of limbal stem cells on polycaprolactone nanofibrous scaffolds for ocular epithelial regeneration. Curr Eye Res, 2016, 41(3): 326-333.

[21] A. Baradaran-Rafii, E. Biazar and S. Heidarikeshel, Cellular response of limbal stem cells on poly(hydroxybuthyrate-co-hydroxyvalerate) porous scaffolds for ocular surface bioengineering. Int. J. Polym Mater Po, 2015, 64: 815-821.

[22] E. Biazar, M.T. Khorasani, N Montazeri, et al., Types of neural guides and using nanotechnology for peripheral nerve reconstruction. Int J Nanomed, 2010, 5: 839-852.

[23] D. Momenzadeh, A. Baradaran-Rafii, K.S. Heidari, et al., Electrospun mat with eyelid fat-derived stem cells as a scaffold for ocular epithelial regeneration. Artif Cell Nanomed B, 2016, 2: 1-8.

[24] R. Ameri, E. Biazar, Development of oriented nanofibrous silk guide for repair of nerve defects. Int. J. Polym Mater Po, 2016, 65(2): 91-95.

[25] A. Bianco, Carbon nanotubes for the delivery of therapeutic molecules. Expert Opin. Drug Delivery, 2004, 1: 57-65.

[26] S.Y. Madani, N. Naderi, O. Dissanayake, et al., A new era of cancer treatment: carbon nanotubes as drug delivery tools. Int. J. Nanomed, 2011, 6: 2963-2979.

[27] S. Prakash, M. Malhotra, W. Shao, et al., Polymeric nanohybrids and functionalized carbon nanotubes as drug delivery carriers for cancer therapy. Adv. Drug Delivery Rev, 2011, 63: 1340-1351.

[28] C. Fabbro, H. Ali-Boucetta, T. Da-Ros, et al., Targeting carbon nanotubes against cancer. Chem. Commun, 2012, 48: 3911-3926.

[29] S.T. Yang, X. Wang, G. Jia, et al., Long-term accumulation and low toxicity of single-walled carbon nanotubes in intravenously exposed mice. Toxicol Lett, 2008, 181: 182-189.

[30] B. Kang, D. Yu, Y. Dai, et al., Cancer-cell targeting and photoacoustic therapy using carbon nanotubes as "bomb" agents. Small, 2009, 5: 1292-1301.

[31] N.W Kam, M. O’Connell, H. Dai, et al., Carbon nanotubes as multifunctional biological transporters and near-infrared agents for selective cancer cell destruction. Proc Natl Acad Sci USA, 2005, 102: 11600-11605.

[32] Y. Xu, M. Mahmood, A. Fejleh, et al., Carbon-covered magnetic nanomaterials and their application for the thermolysis of cancer cell. Int J Nanomedicine, 2010, 5: 167-176.

[33] E.S. Day, L.R. Bickford, J.H. Slater, et al., Antibodyconjugated gold-gold sulfide nanoparticles as multifunctional agents for imaging and therapy of breast cancer. Int J Nanomedicine, 2010, 5: 445-454.

[34] N.W. Kam, H. Dai, Carbon nanotubes as intracellular protein transporters: generality and biological functionality. J Am Chem Soc, 2005, 127: 6021-6026.

[35] D. Borja-Cacho, E.H. Jensen, A.K. Saluja, et al., Molecular targeted therapies for pancreatic cancer. Am J Surg, 2008, 196: 430-441.

[36] L. Mocan, F. Tabaran, T. Mocan, et al., Selective ex-vivo photothermal ablation of human pancreatic cancer with albumin functionalized multivalued carbon nanotubes. Int. J Nanomedicine, 2011, 6: 915-928.

[37] N. Hadidi, F. Kobarfard, N. Nafissi-Varcheh, et al., Optimization of single-walled carbon nanotube solubility by noncovalent PEGylation using experimental design methods. Int J Nanomedicine, 2011, 6: 737-746. 
[38] X. Zheng, F. Zhou. J. Xray, Noncovalent functionalization of single-walled carbon nanotubes by indocyanine green: Potential nanocomplexes for photothermal therapy. J Xray Sci Technol, 2011, 19: 275-284

[39] L. Lacerda, A. Bianco, M. Prato, et al., Carbon nanotubes as nanomedicines: from toxicology to pharmacology. $A d v$. Drug Delivery Rev, 2006, 58: 1460-1470.

[40] A.A. Bhirde, V. Patel, J. Gavard, et al., Targeted killing of cancer cells in vivo and in vitro with EGF-directed carbon nanotube-based drug delivery. ACS Nano, 2009, 3: 307316.

[41] S. Iijima, Helical microtubules of graphitic carbon. Nature, 1991, 354: 56-58.

[42] S. Iijima, Carbon nanotubes: past, present, and future. Phys B Condens Matter, 2002, 323: 1-5.

[43] P.A Tran, L. Zhang and T.J. Webster, Carbon nanofibers and carbon nanotubes in regenerative medicine. Adv. Drug. Delivery Rev, 2009, 61: 1097-1111.

[44] Z. Yang, M. Yang, G. Xiahou, et al., Targeted delivery of insulin-modified immunoliposomes in vivo. J. Liposome Res, 2009, 19: 116-121.

[45] D.K. Chang, C.T. Lin, C.H. Wu, et al., A novel peptide enhances therapeutic efficacy of liposomal anti-cancer drugs in mice models of human lung cancer. PLoS One, 2009, 4: 4171-4182.

[46] J.W. Park, K. Hong, D. B. Kirpotin, et al., Anti-HER2 immunoliposomes: enhanced efficacy attributable to targeted delivery. Clin. Cancer Res, 2002, 8: 1172-1181.

[47] V. Sihorkar, S.P. Vyas, Potential of polysaccharide anchored liposomes in drug delivery, targeting and immunization. J. Pharm. Pharm. Sci, 2001, 4: 138-158.

[48] W. Liang, M. Bockrath, D. Bozovic, et al., Fabry-Perot interference in a nanotube electron waveguide. Nature, 2001, 411: 665-669.

[49] M. Foldvari, M. Bagonluri, Carbon nanotubes as functional excipients for nanomedicines: I. pharmaceutical properties. Nanomedicine, 2008, 4: 173-182.

[50] S. Tavakolifard, E. Biazar, K. Pourshamsian, et al., Synthesis and evaluation of single-wall carbon nanotubepaclitaxel-folic acid conjugate as an anti-cancer targeting agent. Artificial Cells, Nanomedicine, and Biotechnology: An International Journal, 2015: 1-7. Early Online.

[51] R.G. Mendes, A. Bachmatiuk, B. Büchner, et al., Carbon nanostructures as multi-functional drug delivery platforms. J Mater Chem B, 2013, 1: 401.

[52] J.K. Vasir, V. Labhasetwar, Biodegradable nanoparticles for cytosolic delivery of therapeutics. Adv. Drug Delivery Rev, 2007, 59: 718-728.

[53] A.H. Faraji, P. Wipf, Nanoparticles in cellular drug delivery. Bioorg. Med. Chem, 2009, 17: 2950-2962.

[54] R. Siegel, D. Naishadham and A. Jemal, Cancer statistics. Ca-Cancer J. Clin, 2012, 62: 10-29.

[55] S. Dhar, Z. Liu, J. Thomale, et al., Lippard. Targeted single-wall carbon nanotube-mediated Pt (IV) prodrug delivery using folate as a homing device. J. Am. Chem. Soc, 2008, 130: 11467-11476.

[56] S. Aggarwal, Targeted cancer therapies. Nat. Rev. Drug Discov, 2010, 9: 427-428.

[57] H.A. Shih, J.S. Loeffler and N.J. Tarbell, Late effects of CNS radiation therapy. Cancer Treat Res, 2009, 105: 2341.

[58] W.D. Meriwether, N.R. Bachur, Inhibition of DNA and RNA metabolism by daunorubicin and adriamycin in L1210 mouse leukemia. Cancer Res, 1972, 32: 11371142.

[59] C.A Frederick, L.D. Williams and G. Ughetto, et al., Structural comparison of anticancer drug-DNA complexes: adriamycin and daunomycin. Biochemistry, 1990, 29: 2538-2549.

[60] A. Mukherjee, R. Lavery, B. Bagchi, et al., Simulation study of the molecular mechanism of intercalation of the anti-cancer drug daunomycin into DNA. Energy Tran. Dynamic in Biomaterial, 2009, 93: 165-180.

[61] V.P. Torchilin, Recent advances with liposomes as pharmaceutical carriers. Nat. Rev. Drug Discovery, 2005, 4: $145-160$

[62] D. Peer, J.M. Karp, S. Hong, et al., Nanocarriers as an emerging platform for cancer therapy. Nat. Nanotechnol, 2007, 2: 751-760.

[63] R. Sinha, G.J. Kim, S. Nie, et al., Nanotechnology in cancer therapeutics: bioconjugated nanoparticles for drug delivery. Mol. Cancer Ther, 2006, 5: 1909-1917.

[64] M. Grzelczak, M.A. Correa-Duarte, V. SalgueirinoMaceira, et al., Photoluminescence quenching control in quantum dot-carbon nanotube composite colloids using a silica-shell spacer. Adv. Mater, 2006, 18: 415-420.

[65] X. Hong, J. Li, M. Wang, et al., Fabrication of magnetic luminescent nanocomposites by a layer-by-layer selfassembly approach. Chem. Mater, 2004, 16: 4022-4027.

[66] P. Cherukuri, S.M. Bachilo and S.H. Litovsky, Nearinfrared fluorescence microscopy of single-walled carbon nanotubes in phagocytic cells. J. Am. Chem. Soc, 2004, 126: 15638-15639.

[67] M, Dresselhaus, H. Dai, Carbon nanotubes: Continued innovations and challenges. MRS Bull. Adv. Carbon Nanotubes, 2004, 29: 237-243.

[68] K. König, Multiphoton microscopy in life sciences. $J$. Microsc, 2000, 200: 83-104.

[69] S.M. Bachilo, M.S. Strano, C. Kittrell, et al.,Weisman RB. structure-assigned optical spectra of single-walled carbon nanotubes. Science, 2002, 298: 2361-2366.

[70] S. Mukherjee, R.N. Ghosh and F.R. Endocytosis, Physiol. Rev, 1997, 77: 759-803.

[71] Y.W. Cho, J.D. Kim and K. Park, Polycation gene delivery systems: escape from endosomes to cytosol. J. Pharm. Pharmacol, 2003, 55: 721-734.

[72] N. Shao, S. Lu, E. Wickstrom, et al., Integrated molecular targeting of IGF1R and Her2 surface receptors and destruction of breast cancer cells using carbon nanotubes. Nanotechnology, 2007, 18.

[73] E. Basal, G.Z. Eghbali-Fatourechi, K.R. Kalli, et al., Functional folate receptor alpha is elevated in the blood of ovarian cancer patients. PLoS ONE, 2009, 4: e6292

[74] C.J. Mathias, S. Wang, R.J. Lee, et al., Tumor-selective radiopharmaceutical targeting via receptor-mediated endocytosis of gallium-67-deferoxamine-folate. $\mathrm{J} \mathrm{Nucl}$ Med, 1996, 37: 1003-1008.

[75] F. Yang, C. Jin, D. Yang, et al., Magnetic functionalised carbon nanotubes as drug vehicles for cancer lymph node metastasis treatment. European J Cancer, 2011, 47: 18731882.

[76] B. Pauwels, A.E.C. Korst and F. Lardon, Combined modality therapy of gemcitabine and radiation. Oncologist, 2005, 10: 34-51.

[77] F. Yang, D. Fu, J. Long, et al., Magnetic lymphatic targeting drug delivery system using carbon nanotubes. Med Hypotheses, 2008, 70: 765-767.

[78] R. Zboril, M. Mashlan and D. Petridis, Iron(iii) oxides from thermal processes synthesis, structural and magnetic properties, Mossbauer spectroscopy characterization, and applications. Chem Mater, 2002, 14: 969-982.

[79] J. Tucek, R. Zboril and D. Petridis, Maghemite nanoparticles by view of Mossbauer spectroscopy. $J$ Nanosci Nanotechnol, 2006, 6: 926-947.

[80] S. Purushotham, R. Ramanujan, Thermoresponsive magnetic composite nanomaterials for multimodal cancer therapy. Acta Biomater, 2009, 6: 502-510.

[81] S.R. Bhattarai, K.C.R. Badahur, S. Aryal, et al., $\mathrm{N}$-acylated chitosan stabilized iron oxide nanoparticles as a novel nano-matrix and ceramic modification. Carbohydr 
Polym, 2007, 69: 467-477.

[82] B. Chertok, B.A. Moffat, A.E. David, et al., Iron oxide nanoparticles as a drug delivery vehicle for MRI monitored magnetic targeting of brain tumors. Biomaterials, 2008, 29: 487-496.

[83] D. Pantarotto, J.P. Briand, M. Prato, et al., Translocation of bioactive peptidesvacross cell membranes by carbon nanotubes. Chem. Commun, 2004, 1: 16-17.

[84] D. Pantarotto, C.D. Partidos, R. Graff, et al., Synthesis, structural characterization, and immunological properties of carbon nanotubes functionalized with peptides. J. Am. Chem. Soc, 2003, 125: 6160-6164.

[85] N.W.S. Kam, T.C. Jessop, P.A. Wender, et al., Nanotube molecular transporters: internalization of carbon nanotube protein conjugates into mammalian cells. J. Am. Chem. Soc, 2004, 126: 6850-6851.

[86] E. Smythe, Clathrin-coated vesicle formation: a paradigmfor coated-vesicle formation. Biochem. Soc. Trans, 2003, 31: 736-739.

[87] N.W.S. Kam, Z.L.H. Dai, Carbon nanotubes as intracellular transporters for proteinsand DNA: an investigation of the uptake mechanism and Pathway. Ang. Chem. Int. Edition, 2006, 45: 577-581.

[88] B. Kang, S. Chang, Y. Dai, et al., Cell response to carbon nanotubes: Size-dependent intracellular uptake mechanism and subcellular fate. Small, 2010, 6: 23622366.

[89] S.K. Smart, A.I. Cassady, G.Q. Lu, et al., The biocompatibility of carbon nanotubes. Carbon, 2006, 44: 1034-1047.

[90] R. Hirlekar, M. Yamagar, H. Garse, et al., Carbon nanotubes and its applications: a review. Asian J. Pharm. Clin, 2009, 2: 17-27.

[91] C.F. Lopez, S.O. Nielsen and P.B. Moore, Understanding nature's design for a nanosyringe. Proc. Natl. Acad. Sci. U.S.A, 2004, 101: 4431-4434.

[92] Z. Liu, S. Tabakman, K. Welsher, et al., Carbon nanotubes in biology and medicine: in vitro and in vivo detection, imaging and drug delivery. Nano Res, 2009, 2: 85-120.

[93] D. Tasis, N. Tagmatarchis, V. Georgakilas, et al., Soluble carbon nanotubes. Chem. A Eur. J, 2003, 9: 4000-4008.

[94] V.L. Colvin, The potential environmental impact of engineered nanomaterials. Nat. Biotech, 2003, 21: 11661170.

[95] D.B. Warheit, B.R. Laurence, K.L. Reed, et al., Comparative pulmonary toxicity assessment of singlewall carbon nanotubes in rats. Toxicol. Sci, 2004, 77: 117125.

[96] A. Bianco, K. Kostarelos, C.D. Partidos, et al., Biomedical applications of functionalized carbon nanotubes. Chem. Commun, 2005, 5: 571-577.

[97] J.L. Bahr, E.T. Mickelson, M.J. Bronikowski, et al., Dissolution of small diameter single-wall carbon nanotubes in organic solvents. Chem. Commun, 2001, 2: 193-194.

[98] M.J. O’Connell, P. Boul, L.M. Ericson, et al., Reversible water-solubilization of single-walled carbon nanotubes by polymer wrapping. Chem. Phys. Lett, 2001, 342: 265-271.

[99] J. Chen, H. Liu, W.A. Weimer, et al., Noncovalent engineering of carbon nanotube surfaces by rigid, functional conjugated polymer. J. Am. Chem. Soc, 2002, 124: 9034-9035.

[100] M. Zheng, A. Jagota and M.S.M. Strano, Structure-based carbon nanotube sorting by sequence-dependent DNA assembly. Science, 2003, 302: 1545-1548.

[101] S. Bandow, A.M. Rao, K.A. Williams, et al., Purification of single-wall carbon nanotubes by microfiltration. $J$. Phys. Chem. B, 1997, 101: 8839-8842.

[102] M. Adeli, R. Sepahvand, B. Astinchap, et al., Carbon nanotube-graft-block copolymers containing silver nanoparticles. Int. J. Nano Sci, 2009, 8: 533-541.

[103] M. Adeli, A. Bahari and H. Hekmatara, Carbon nanotubegraft-poly-(citric acid) nanocomposites. Nano, 2008, 3: 37-44.

[104] M. Adeli, N. Mirab, M.S. Alavidjeh, et al., Carbon nanotubes-graft-polyglycerol: Biocompatible hybrid materials for nanomedicine. Polymer, 2009, 50: 35283536.

[105] M. Adeli, S. Beyranvand and M. Hamid, Noncovalent interactions between linear-dendritic copolymers and carbon nanotubes lead to liposome-like nanocapsule. $J$. Mater. Chem, 2012, 22: 6947-6952.

[106] F. Yuan, M. Dellian, D. Fukumura, et al., Vascular permeability in a human tumor xenograft: molecular size dependence and cutoff size. Cancer Res, 1995, 55: 37523756.

[107] S.K. Hobbs, W.L. Monsky, F. Yuan, et al., Regulation of transport pathways in tumor vessels: role of tumor type and microenvironment. Proc. Natl. Acad. Sci. U.S.A, 1998, 95: 4607-4612.

[108] G. Pastorin, W. Wu, S. Wieckowski, et al., Double functionalisation of carbon nanotubes for multimodal drug delivery. Chem. Commun, 2006, 11: 1182-1184

[109] Z. Liu, K. Chen, C. Davis, et al., Drug delivery with carbon nanotubes for in vivo cancer treatment. Cancer Res, 2008, 68: 6652-6660.

[110] Z. Liu, X. Sun, N.R Nakayama, et al., Supramolecular chemistry on water-soluble carbon nanotubes for drug loading and delivery. ACS Nano, 2007, 1: 50-56.

[111] Z. Ji, G. Lin, Q. Lu, et al., Targeted therapy of SMMC7721 liver cancer in vitro and in vivo with carbon nanotubes based drug delivery system. J. Colloid and Interface Science, 2012, 365: 143-149

[112] X. Zhang, L. Meng, Q. Lu, et al., Targeted delivery and controlled release of doxorubicin to cancer cells using modified single wall carbon nanotubes. Biomaterials, 2009, 30: 6041-6047.

[113] P. Jeyamohan, T. Hasumura, Y. Nagaoka, et al., Accelerated killing of cancer cells using a multifunctional single-walled carbon nanotube-based system for targeted drug delivery in combination with photothermal therapy. Int. J. Nanomedicine, 2013, 8: 2653-2667.

[114] Z. Tian, Y. Shi, M. Yin, et al., Functionalized multivalued carbon nanotubes anticancer drug carriers: synthesis, targeting ability and antitumor activity. Nano Biomed Eng, 2011, 3: 157-162.

[115] M. Adeli, F. Hakimpoor, M. Ashiri, et al., Anticancer drug delivery systems based on noncovalent interactions between carbon nanotubes and linear-dendritic copolymers. Soft Matter, 2011, 7: 4062-4070.

[116] Z. Sobhani, R. Dinarvand, F. Atyabi, et al., Increased paclitaxel cytotoxicity against cancer cel lines using a novel functionalized carbon nanotube. Int. J. Nanomedicine, 2011, 6: 705-719.

[117] J. Chen, S. Chen, X. Zhao, et al., Functionalized singlewalled carbon nanotubes as rationally designed vehicles for tumor-targeted drug delivery. J. Am. Chem. Soc, 2008, 130: 16778-16785.

[118] E. Heister, V. Neves, C. Tilmaciu, et al., Triple functionalization of single-walled carbon nanotubes with doxorubicin, a monoclonal antibody, and a fluorescent marker for targeted cancer therapy. Carbon, 2009, 47: 2152-2160

[119] X. Lou, R. Daussin, S. Cuenot, et al., Synthesis of pyrene-containing polymers and noncovalent sidewall functionalization of multiwalled carbon nanotubes. Chem. Mater, 2004, 16: 4005-4011.

[120] S. Detriche, S. Devillers, J.F. Seffer, et al., The use of water-soluble pyrene derivatives to probe the surface of carbon nanotubes. Carbon, 2011, 49: 2935-2943. 
[121] C. Klumpp, K. Kostarelos, M. Prato, et al., Functionalized carbon nanotubes as emerging nanovectors for the delivery of therapeutics. Biochim Biophys Acta, 2006, 1758: 404-412

[122] M. Bottini, N. Rosato and N. Bottini, PEG-modified carbon nanotubes in biomedicine: current status and challenges ahead. Bio macromolecules, 2011, 12: 33813393.

[123] L. Vaisman, H.D. Wagner amd G. Marom, The role of surfactants in dispersion of carbon nanotubes. Adv Colloid Interface Sci, 2006, 128: 37-46.

[124] X. Liu, H. Tao, K. Yang, et al., Optimization of surface chemistry on single-walled carbon nanotubes for in vivo photothermal ablation of tumors. Biomaterials, 2011, 32: 144-151.

[125] Z. Liu, M. Winters, M. Holodniyet, et al., siRNA delivery into human $\mathrm{T}$ cells and primary cells with carbonnanotube transporters. Angew Chem Int Ed, 2007, 46: 2023-2027.

[126] N.R. Nakayama, S. Bangsaruntip, X.M. Sun, et al., Noncovalent functionalization of carbon nanotubes by fluorescein-polyethylene glycol: Supramolecular conjugates with pH-dependent absorbance and fluorescence. J. Am. Chem. Soc, 2007, 129: 2448-2449.

[127] R. Chen, Y. Zhang, H. Dai, et al., Noncovalent sidewall functionalization of single-walled carbon nanotubes for protein immobilization. J. Am. Chem. Soc, 2001, 123: 3838-3839.

[128] Q.W. Li, B.Q. Sun, I.A. Kinloch, et al., Sirringhaus H, Windle AH. Enhanced self-assembly, of pyridine-capped CdSe nanocrystals on individual single-walled carbon nanotubes. Chem. Mater, 2006, 18: 164-168.

[129] P.A. Allen, W. Liu, V.P. Chauhan, et al., InAs(ZnCdS) quantum dots optimized for biological imaging in the near-infrared. J. Am. Chem. Soc, 2010, 132: 470-471.

[130] B. Kang, D.C. Yu, S.Q. Chang, et al., Intracellular uptake, trafficking and subcellular distribution of folate conjugated single walled carbon nanotubes within living cells. Nanotechnology, 2008, 19: 375103.

[131] C. Fu, L. Meng, Q. Lu, et al., Large-scale production of homogeneous helical amylose/SWNTs complexes with good biocompatibility. Macromol Rapid Commun, 2007, 28: 2180-2184.

[132] M. Numata, M. Sai, A.K. Kaneko, et al., Curdlan and schizophyllan (beta-1,3-glucans) can entrap single-wall carbon nanotubes in their helical superstructure. Chem Lett, 2004, 33: 232-233.

[133] I. Gurevitch, S. Srebnik, Monte Carlo simulation of polymer wrapping of nanotubes. Chem Phys Lett, 2007, 444: $96-100$.

[134] V.G.S. Box, The intercalation of DNA double helices with doxorubicin and nagalomycin. J Mol Graph, 2007, 26: 14-19.

[135] S.M. Zeman, D.R. Phillips and D.M. Crothers, Characterization of covalent Adriamycin DNA adducts. Proc Natl Acad Sci, 1998, 95: 11561-11565.

[136] M. Adeli (Ed.), Cancer etiology, diagnosis and treatments: Hybrid nanostructures in cancer therapy. Nova Science Publishers, 2012: 214.

[137] M.J. O>Connell, S.H. Bachilo, C.B. Huffman, et al., Band gap fluorescence from individual single-walled carbon nanotubes. Science, 2002, 297: 593-596.

[138] J.L. Eiseman, N.D. Eddington, J. Leslie, et al., Plasma pharmacokinetics and tissue distribution of paclitaxel in CD2F1 mice. Cancer Chemother Pharmacol, 1994, 34: 465-471.

[139] A. Sparreboom, O.T. van, W.J. Nooijen, Tissue distribution, metabolism and excretion of paclitaxel in mice. Anticancer Drugs, 1996, 7: 78-86.

[140] A. Gangloff, W.A. Hsueh, A.L. Kesner, et al., Estimation of paclitaxel biodistribution and uptake in human-derived xenografts in vivo with (18) F-fluoropaclitaxel. $\mathrm{J} \mathrm{NuCl}$ Med, 2005, 46: 1866-1871.

[141] A. Sparreboom, O.T. Van, W.J. Nooijen, et al., Nonlinear pharmacokinetics of paclitaxel in mice results from the pharmaceutical vehicle Cremophor EL. Cancer Res, 1996, 56: 2112-2115.

[142] T.K. Yeh, Z. Lu, M.G. Wientjes, et al., Formulating paclitaxel in nanoparticles alters its disposition. Pharm Res, 2005, 22: 867-874.

[143] X.Y. Chen, C. Plasencia, Y.P. Hou, et al., Synthesis and biological evaluation of dimeric RGD peptide-paclitaxel conjugate as a model for integrin-targeted drug delivery. $J$ Med Chem, 2005, 48: 1098-1106.

[144] J. Nakamura, N. Nakajima, K. Matsumura, et al., In vivo cancer targeting of water-soluble taxol by folic acid immobilization. Journal Nanomedic Nanotechnol, 2011, 2: 106.

[145] E. Lee, J. Lee, L. In-Hyun, et al., Conjugated chitosan as a novel platform for oral delivery of paclitaxel. J. Med. Chem, 2008, 51: 6442-6449.

[146] K. Kostarelos, A. Bianco, M. Prato, et al., Promises, facts and challenges for carbon nanotubes in imaging and therapeutics. Nat. Nanotechnol, 2009, 4: 627-633.

[147] H.H.P. Yiu, Engineering the multifunctional surface on magnetic nanoparticles for targeted biomedical applications: A chemical approach. Nanomedicine, 2011, 6: 1429-1446.

[148] S. Cheong, P. Ferguson, K.W. Feindel, et al., Simple synthesis and functionalization of iron nanoparticles for magnetic resonance imaging. Angew. Chem. Int. Ed, 2011, 50: 4206-4209.

[149] J. Dobson, Magnetic micro- and nano-particle-based targeting for drug and gene delivery. Nanomedicine, 2006, 1: 31-37.

[150] J. Park, G. Maltzahn, E. Ruoslahti, et al., Micellar hybrid nanoparticles for simultaneous magnetofluorescent imaging and drug delivery. Angew. Chem., Int. Ed, 2008, 47: 7284-7288.

[151] N. Cho, T. Cheong, J. HyunMin, et al., A multifunctional core-shell nanoparticle for dendritic cell-based cancer immunotherapy. Nat. Nanotechnol, 2011, 6: 675-682.

[152] O. Metin, V. Mazumder, S. Ozkar, et al., Monodisperse nickel nanoparticles and their catalysis in hydrolytic dehydrogenation of ammonia borane. J. Am. Chem. Soc. 2010, 132: 1468-1469.

[153] M.A. Gonzalez-Fernandez, T.E. Torres, M. Andres-Verges, et al., Magnetic nanoparticles for power absorption: Optimizing size, shape and magnetic properties. J. Solid State Chem, 2009, 182: 2779-2784.

[154] D. Ugarte, A. Chatelain and W.A. De-Heer, Nanocapillarity and chemistry in carbon nanotubes. Science, 1996, 274: 1897-1899.

[155] P.M. Ajayan, T.W. Ebbesen, S Iijima, et al., Opening carbon nanotubes with oxygen and implications for filling. Nature, 1993, 362: 522-525.

[156] K. Svensson, H. Olin and E. Olsson, Nanopipettes for metal transport. Phys. Rev. Lett, 2004, 93: 145901145903.

[157] H. He, Y. Zhang, C. Gao, et al., "Clicked” magnetic nanohybrids with a soft polymer interlayer. Chem. Commun, 2009, 13: 1655-1657.

[158] K. Jiang, A. Eitan, L.S. Schadler, et al., Selective attachment of gold nanoparticles to nitrogen-doped carbon nanotubes. Nano Lett, 2003, 3: 275-277.

[159] Z. Wang, M. Li, Y. Zhang, et al., Thionine-interlinked multi-walled carbon nanotube/gold nanoparticle composites. Carbon, 2007, 45: 2111-2115.

[160] G.M. Rahman, D.M. Guldi, E. Zambon, et al., Dispersable carbon nanotube/gold nanohybrids: Evidence for strong 
electronic interactions. Small, 2005, 1: 527-530.

[161] C. Wang, C. Bao, S. Liang, et al., RGD-conjugated silicacoated gold nanorods on the surface of carbon nanotubes for targeted photoacoustic imaging of gastric cancer. Nanoscale Res. Lett, 2014, 9: 264.

[162] B.S. Gutrath, M.F. Beckmann, A. Buchkremer, et al., Size-dependent multispectral photoacoustic response of solid and hollow gold nanoparticles. Nanotechnology, 2012, 23: 225707.

[163] D.P. Yang, D.X. Cui, Advances and prospects of gold nanorods. Chem Asian J, 2008, 12: 2010-2022.

[164] C. Bao, N. Beziere, P. del Pino, et al., Gold nanoprisms as optoacoustic signal nanoamplifiers for in vivo bioimaging of gastrointestinal cancers. Small, 2013, 9: 68-74.

[165] C. Wang, Z.M. Li, B. Liu, et al. Dendrimer modified SWCNTs for high efficient delivery and intracellular imaging of surviving siRNA. Nano Biomed Eng, 2013, 5: 125-130.

[166] H Gong, R Peng and Z Liu. Carbon nanotubes for biomedical imaging: the recent advances. Adv Drug Deliv Rev, 2013, 65(15): 1951-1963.

[167] J.W. Kim, E.I. Galanzha, E.V. Shashkov, et al., Golden carbon nanotubes as multimodal photoacoustic and photothermal high contrast molecular agents. Nat Nanotechnol, 2009, 4: 688-694.

[168] D. Yang, F. Yang, J.H. Hu, et al., Hydrophilic multi-walled carbon nanotubes decorated with magnetite nanoparticles as lymphatic targeted drug delivery vehicles. Chem Commun, 2009, 29: 4447-4449.

[169] D. Cai, J.M. Mataraza, Z. Qin, et al., Highly efficient molecular delivery into mammalian cells using carbon nanotube spearing. Nat. Methods, 2005, 2: 449-454.

[170] E.C. Vermisoglou, G. Pilatos, G.E. Romanos, et al., Magnetic carbon nanotubes with particle-free surfaces and high drug loading capacity. Nanotechnology, 2011, 22: 35602-35611.

[171] X. Yang, X. Zhang, Y. Ma, et al., Superparamagnetic graphene oxide- $\mathrm{Fe}_{3} \mathrm{O}_{4}$ nanoparticles hybrid for controlled targeted drug carriers. J. Mater. Chem, 2009, 19: 27102714.

[172] M.L. Chen, Y.J. He, X.W, et al., Chen, quantum dots conjugated with $\mathrm{Fe}_{3} \mathrm{O}_{4}$ filled carbon nanotubes for cancertargeted imaging and magnetically guided drug delivery. Langmuir, 2012, 28: 16469-16476.

[173] J. Ai, E. Biazar, M. Jafarpour, et al., Nanotoxicologynanoparticles safety at biomedical designs. Int. J. Nanomed, 2011, 6: 1117-1127

[174] J.J. Khandare, A. Jalota-Badhwar, S.D. Satavalekar, et al., PEG-conjugated highly dispersive multifunctional magnetic multi-walled carbon nanotubes for cellular imaging. Nanoscale, 2012, 4: 837-844.

[175] A. Masotti, A. Caporali, Preparation of magnetic carbon nanotubes (Mag-CNTs) for biomedical and biotechnological applications. Int. J. Mol. Sci, 2013, 14: 24619-24642.

[176] M. Horie, H. Kato, K. Fujita, et al., In vitro evaluation of cellular response induced by manufactured nanoparticles. Chem Res Toxicol, 2012, 25: 605-619.

[177] K. Kostarelos, Rational design and engineering of delivery systems for therapeutics: biomedical exercises in colloid and surface science. Adv. Colloid Interface Sci, 2003, 106: 147-168.

[178] A. Nel, T. Xia, L. Maedler, et al., Toxic potential of materials at the nano level. Science, 2006, 311: 622-627.

[179] K. Donaldson, R. Aitken, L. Tran, et al., Carbon nanotubes: a review of their properties in relation to pulmonary toxicology and workplace safety. Toxicol Sci, 2006, 92: 5-22.

[180] R.J. Toh, A. Ambrosi and M. Pumera, Bioavailability of metallic impurities in carbon nanotubes is greatly enhanced by ultrasonication. Chemistry, 2012, 18: 1159311596.

[181] S. Pichardo, D. Gutierrez-Praena, M. Puerto, et al., Oxidative stress responses to carboxylic acid functionalized single wall carbon nanotubes on the human intestinal cell line Caco-2. Toxicol In Vitro, 2012, 26: 672677.

[182] A. Hirsch, Functionalization of single-walled carbon nanotubes. Angew. Chem., Int. Ed, 2002, 41: 1853-1859.

[183] K. Balasubramanian, M. Burghard, Chemically functionalized carbon nanotubes. Small, 2005, 1: 180-192.

[184] Z. Liu, C. Davis, W. Cai, et al., Circulation and long-term fate of functionalized, biocompatible single-walled carbon nanotubes in mice probed by Raman spectroscopy. Proc. Natl. Acad. Sci. U.S.A, 2008, 105: 1410-1415

[185] D. Cui, F. Tian, C.S. Ozkan, et al., Effect of single wall carbon nanotubes on human HEK293 cells. Toxicology Letters, 2005, 155: 73-85.

[186] H. Gao, Y. Kong, D. Cui, et al., Spontenous insertion of DNA oligonucleotides into carbon nanotubes. Nano Letters, 2003, 3: 471-473.

[187] F. Tian, D. Cui, H. Schwarz, et al., Cytotoxicity of singlewall carbon nanotubes on human fibroblasts. Toxicology In Vitro, 2006, 20: 1202-1212.

[188] D. Cui, Advances and prospects on biomolecules functionalized carbon nanotubes. J. Nanosci and Nanotechnology, 2007, 7: 1298-1314.

Copyright $(\subset 2016$ Sara Tavakolifard, Esmaeil Biazar. This is an open-access article distributed under the terms of the Creative Commons Attribution License, which permits unrestricted use, distribution, and reproduction in any medium, provided the original author and source are credited. 